\title{
Adaptation to displaced vision measured with three tests'
}

OLIVIA SHAFFER AND HANS WALLACH

SWARTHMORE COLLEGE

Adaptation to laterally displacing prisms was produced by walking a narrow hallway for $1 / 2 \mathrm{hr}$. Three tests given before and after adaptation showed a significant adaptation effect. Pointing to a visual target and pointing straight ahead in darkness showed apparently equal adaptation, while a visual forward direction test measured a smaller effect.

Adaptation to wedge prisms which displace the visual direction laterally has been studied under a variety of conditions. Five different specific training conditions have been employed: walking (Held \& Bossom, 1961), regarding one's moving arm while seated (Held \& Hein, 1958), looking at one's legs while standing still or lying supine (Wallach et al, 1963) and pointing repeatedly at a target (Harris, 1963). Three types of tests have been used: pointing at a target under conditions that kept the pointing arm hidden (PT), pointing straight ahead in total darkness (PSA), and a visual forward direction test (FD) used in two different versions; $S$ who was seated on a rotating chair had to turn himself to a position where a target appeared straight ahead (Held \& Bossom, 1961), and S with head position fixed by a bite board directed a movable target to appear straight ahead (Wallach etal, 1963). Never have all three types of tests been used in connection with one training condition. Harris (1963) used PT and PSA in connection with training by repeated pointing but, for good reason, did not use FD. Wallach et al (1963) used PT and FD in connection with looking at one's legs and obtained inconsistent results. When $\mathrm{S}$ stood during training, PT and FD showed equal adaptation effects. When training was supine, FD showed a large amount of adaptation, while PT showed no effect at all. It seemed appropriate to employ all three types of tests in connection with the same training condition. We selected walking as our training condition.

Procedure

The three tests were given without prisms before and after adaptation training. S's left eye was occluded with a patch during the tests. The tests were given in the dark and S's eyes were closed except when a response required looking at the target. The apparatus used was described in Wallach et al (1963). Briefly, S stood with his teeth in a mold above the center of a horizontal board which could be raised or lowered. A rim on the far edge of the board served as a rail for a sliding fixture which carried a flashlight bulb. The bulb was dimly lit for use as a target in the pointing-to-target test and the forward-direction test. A measuring stick attached to the far edge of the board was used to read the position of S's pointing finger and of the target. For the PT test, $E$ set the target $7^{\circ}$ to the left of $S^{\prime} s$ median plane. E asked $S$ to open his eyes and point to the target, close his eyes and maintain the position of his arm. E then read the position of S's pointing finger with a flashlight. For the FD test, two settings were taken, one starting from the left and one from the right. E placed the target clearly too far to one side before asking $S$ to open his eyes, then told $S$ to indicate when the target appeared straight ahead. E moved the target towards the center in discrete steps, asking $S$ whether to move it more in the same direction after each step. $S$ answered by means of prearranged grunting signals (as $S$ could not speak with his mouth in the mold). If $S$ answered "no," $E$ asked whether the target should be moved back in the other direction before asking whether it was now straight ahead. The size of the steps decreased as S'S judgment became more hesitant. Since E could not read the position of the target in the dark, no bias was possible despite the informality of the procedure. After a forward direction setting was completed in one direction, $E$ read the result with the flashlight and then began a setting in the other direction. For the PSA test, $\mathrm{S}$ was instructed, "Point straight ahead of you, keeping your eyes closed." When $\mathrm{S}$ indicated he was pointing straight ahead, $E$ read the position of his pointing finger.

When the three initial tests were completed, a 20 diopter prism was put in front of S's right eye. The prism was attached to the right-eye opening of welder's goggles from which the glasses had been removed. The left-eye opening of the goggles was covered. The outside surface of the prism was covered by a Wratten Filter 58 which eliminated the colored fringes that the prism ordinarily produced. S's eyes were always closed while wearing the prism in the testing room. E led $\mathrm{S}$ to a narrow hallway $55 \mathrm{ft}$. long and $5 \mathrm{ft}$. wide. The hallway contained a number of doors, some of which were open. $S$ then opened his eyes and walked back and forth along the hallway, with E's aid at first, thereafter by himself. $S$ was told never to look down, since it was desirable to avoid confounding this adaptation procedure with the legviewing procedure of Wallach et al. After $30 \mathrm{~min}$. of walking, $\mathrm{S}$ closed his eyes and $\mathrm{E}$ removed the goggles and brought him back to the testing table for a repetition of the tests.

Twenty-four undergraduates were employed. Twelve Ss wore a right-displacing prism and 12 a left-displacing prism. For a given $S$, the order of the three tests was the same before and after walking. There are six orders in which three tests may be given and, in addition, the 
forward direction test may be given in two ways (starting with determination of the left or the right limit), making a total of 12 possible orders of tests. For each direction of displacement, all 12 possible orders were given.

\section{Results}

For each $S$ on each test the difference between the post-adaptation score and the pre-adaptation score was calculated. Differences in the adaptive direction were considered positive. For the FD test the adaptation score was the average of the two differences obtained (one for the left and one for the right limit). Since there was no effect of test order apparent, the statistical analysis did not take test order into account.

For the left and right prisms combined, the average adaptation was $4^{\circ} 29^{\prime}$ on PT, $4^{\circ} 37^{\prime}$ on PSA and $2^{\circ} 3^{\prime}$ on FD. Tests demonstrated that all these effects were significant at the .02 level or better (see Table 1). Although mean adaptation was virtually identical for PSA and PT, PSA scores were more variable than PT scores. The difference in variance was significant both for the left prism $(F=4.63, p<.01)$ and for the right prism $(F=3.87, p<.05)$.

An analysis of variance was performed on the PT and the FD scores. PSA scores were not included in this analysis because of their large variance. The analysis revealed that the difference between tests was significant $(F=6.00, \mathrm{df}=1 / 22, \mathrm{p}<.05)$. Although the left-displacing prism produced a larger effect on all tests, the difference between these effects was not significant; neither was the interaction between tests and displacement direction significant.

\section{Discussion}

The results show that $1 / 2 \mathrm{hr}$. of walking while wearing a laterally-displacing prism produces effects on all

Table 1. Adaptation as Measured by each Test

\begin{tabular}{llcc} 
Test & Prism & $\begin{array}{c}\text { Mean } \\
\text { Adaptation }\end{array}$ & $P$ \\
\hline \multirow{3}{*}{ PT } & Left & $5^{\circ} 41^{\prime}$ & $<.01$ \\
& Right & $3^{\circ} 7^{\prime}$ & $<.02$ \\
& Both & $4^{\circ} 29^{\prime}$ & $<.001$ \\
FD & Left & $2^{\circ} 13^{\prime}$ & $<.10$ \\
& Right & $1951^{\prime}$ & $>.10$ \\
& Both & $2^{\circ} 3^{\prime}$ & $<.02$ \\
PSA & Left & $5^{\circ} 35^{\prime}$ & $<.02$ \\
& Right & $3^{\circ} 39^{\prime}$ & $>.10$ \\
& Both & $4^{\circ} 37^{\prime}$ & $<.01$ \\
\hline
\end{tabular}

three tests of adaptation, PT, PSA, and FD. The effects measured with the PT and the PSA tests were apparently equal, while FD measured a smaller mean effect than PT. The difference between the FD and PT tests shows that these tests must measure, at least in part, intrinsically different effects. We consider this the main result of our experiment. A dissociation between results of FD and PT tests first found by Wallach et al was once again obtained. If we could accept as an established fact the apparent result that the effects measured by PT and PSA are of the same magnitudea finding which cannot be statistically justified because of the high variability of the PSA scores-a further conclusion could be drawn. Since the perceptual tasks on the PT and PSA tests differ while the responses are the same, one could conclude that the adaptation effect derived from walking, which is measured by these tests, is, at least in part, peculiar to the pointing response. Such a pattern of results could be easily understood if the training situation required $S$ to move his arm and look at it (Harris, 1963), but was not expected in our experiment, where training was by walking. The conclusion that walking per se produces adaptation peculiar to the pointing response is supported by a result of Hay \& Pick (1966), which was unknown to us at the time our experiment was performed. Hay and Pick had their Ss point to a visual as well as to an auditory target. When the training conditions were similar to ours, the two pointing tests showed an equal amount of adaptation.

\section{References}

Hay, J. C., \& Pick, H. L.. Jr. Visual and proprioceptive adaptation to optical displacement of the visual stimulus. $J$. exp. Psychol., 1966, 71, 150-158.

Harris, C. S. Adaptation to displaced vision: visual, motor, or proprioceptive change? Science, 1963, 140, 812-813.

Held, R., \& Bossom, Jr. Neonatal deprivation and adult rearrangement. J. comp. physiol. Psychol., 1961, 54, 33-37.

Held, R., \& Hein, A. V. Adaptation of disarranged hand-eye coordination contingent upon reafferent stimulation. Percept. mot. Skills, $1958,8,87-90$.

Wallach, H., Kravitz, J. H., \& Lindauer, Judith. A passive condition for rapid adaptation to displaced visual direction. Amer. J. Psychol., 1963, 76, 568-578.

\section{Note}

1. This work was supported by a grant from the National Science Foundation. 